Volume 1 | No. 2 | July - December 2017

\title{
Theoretical Insights into Coverage Analysis of Cellular Networks
}

Murk Marvi $^{1}$, Muhammad Khurram ${ }^{1}$

\begin{abstract}
:
Recently tools from stochastic geometry have gained much attention for modeling and analysis of dense cellular networks. Although extensive studies are available in literature in this respect, the approaches are generalized and often lack significance towards practical scenarios where network conditions vary dynamically. The main objective of this research is to provide some insights into the stochastic geometry based analysis of cellular networks through suitable modifications so that it can be used to estimate parameters of interest i.e., intensity and coverage probability for different tiers and scenarios under consideration. The main definition for probability of coverage has been re-defined and complete closed form expression is derived. The intensity for different tiers have been estimated with the help of proposed approach, beyond which no more gain in coverage probability can be achieved. Monte-Carlo simulations have been performed for validation of proposed approach and results are also compared with stateof-the-art approach.
\end{abstract}

Keywords: Stochastic geometry, Coverage analysis, Cellular network

\section{Introduction}

The rapid development in handheld digital devices i.e., smartphones, tablets, laptops, and machine type communication have resulted in exponential growth for capacity requirements. Apart from this, explosion of emerging resource hungry services and applications are playing a dominating role in overall increase in traffic demands. The traditional cellular networks were designed to provide simple voice communication ( $1 \mathrm{G}$ and $2 \mathrm{G}$ ) after that enhanced to provide data communication as well (beyond 2G) to its customers. Initially, the network users were only humans; however, in current scenario, the network has to serve machine type applications along with conventional human-to-human applications. Therefore, existing cellular network is not capable of fulfilling the growing demands with extreme variability in service requirements like delay, throughput, packet loss and other related metrics of measure. Thus, the concept of dense heterogeneous i.e., multi-tier networks [1]-[3] has been exploited in literature for fulfilling capacity requirements of customers. In traditional network. Since the demands were low, the focus was around coverage analysis only. However, in existing networks the focus has been shifted to capacity analysis as well in order to meet the requirements of customers and that's the main reason behind coined concept of dense networks. As ergodic capacity of a network is limited due to interference experienced by the customers which is random; hence, it is important to analyze the distribution of this random variable across the network.

\subsection{Related Work}

Traditionally, Wyner [4] and grid [5] models have been employed for estimating coverage and capacity bounds in cellular networks. Wyner model provides good insight into high interference regime [6], however in general it is considered to be highly inaccurate. On the other hand, grid model considers regular deployment of base stations (BSs) which is, practically, not the case especially for dense networks. Therefore, grid model provides upper bound to interference

${ }^{1}$ Computer \& Information Systems Department, NED University of Engineering \& Technology, Pakistan

Corresponding email:* marvi@ neduet.edu.pk

SJCMS | P-ISSN: 2520-0755 | E-ISSN: 2522-3003 @ 2017 Sukkur IBA University - All Rights Reserved 
distribution. Recently stochastic geometry has gained much attention for coverage and rate analysis of dense cellular networks due to its tractability for special cases and simple numerical integrations for general cases. In [7], a tractable approach has been presented for coverage and rate analysis of single-tier cellular network by modeling the location of BSs through a Poisson point process (PPP). The results provided in [7] are pessimistic due to presence of strong interfering nodes nearby; they are extremely generalized and tractable as well. In [8], coverage and rate analysis for multi-tier network have been presented by exploiting max SINR BS association rule. On the other hand in [9], same analysis has been carried out as in [8], but nearest BS association rule is used. The interesting finding in [8], [9] is that, for interference limited network with Rayleigh fading and path loss exponent of 4, the probability of coverage for multi-tier cellular network becomes equivalent to that of single-tier case presented in [7]. The authors have justified this result by making an argument that addition of low power tiers into existing dense Macro tier will not affect the coverage in any case. Since any increase in interference will be counterbalanced by increase in received signal strength, additional BSs can be deployed for achieving linear increase in capacity of the network without affecting the coverage. Due to pessimistic results provided by PPP model, in [10] detrimental point process (DPP) is used where correlation or repulsive effect has been taken into account. Therefore, authors in [10] have claimed that DPP provides better coverage and rate analysis as compared to PPP. Similarly in [11], Poisson cluster process (PCP) has been exploited by considering the fact that certain regions are denser as compared to the others. The authors in [11] have compared to the results of PCP with PPP and concluded that in PPP capacity increases linearly with increase in number of tiers; whereas in PCP the coverage and capacity degrades as number of tiers grow beyond certain threshold. In [12], spatial dependence for BSs deployment have been considered for two tier network i.e., Macro and Pico, where the locations of Macro BSs have been drawn from a PPP and for Pico BSs Poisson hole process (PHP) has been used. The authors have verified results through simulation and claimed that proposed numerical results are closely fitting to the simulation results. The approaches proposed by [7]-[9] are pessimistic; however, they are tractable under especial cases. On the other hand, approaches proposed in [10]-[12] involve numerical integration but provide better approximation to actual scenarios.

Recently, in [13] the researchers have discussed fundamental limits on wireless network densification. Over the past few years, the concept of densification has been exploited for enhancing data rates. The researchers in [13] have argued that at some point further densification will no longer be able to provide exponentially increasing data rates like Moore's law. Traditionally, simple path loss model is used for coverage analysis. However, recently researchers have exploited multi-slope path loss models for better coverage analysis of dense cellular networks [14]. Authors have suggested that, due to densification, the distance between customer and $\mathrm{BS}$ is less than certain critical distance

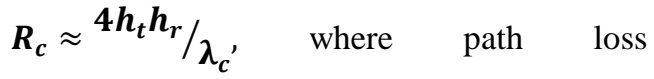
exponent $\boldsymbol{\alpha} \approx 2$. In general, for distance greater than $\boldsymbol{R}_{\boldsymbol{c}}$ the path loss exponent $\boldsymbol{\alpha} \approx \mathbf{4}$. Thus, with the help of multi-slope path loss model coverage probability can be more accurately estimated. In [14], the authors have used dual slope path loss model and showed that as network density grows and $\boldsymbol{\alpha}<\mathbf{1}$, potential throughput approaches to zero. On the other hand, for $\boldsymbol{\alpha}>\mathbf{1}$ potential throughput grows with denser deployment. However, growth may be sublinear depending on the path loss exponent.

\subsection{Motivation and Contribution}

Even though extensive studies are available in literature for coverage and rate 
analysis of cellular networks by exploiting tools from stochastic geometry; such approaches are extremely generalized and cannot be used for analyzing specific network scenarios, which are more useful for industry practitioners. For example, in [7]-[9] tractable models have been presented for coverage analysis of dense single and multi-tier cellular network, which are independent of power and intensity of BSs under special conditions; however, few questions mentioned here are really important to answer from practical perspective, 1) What is the limit of $\boldsymbol{\lambda}$, for particular tier, beyond which it can be considered as dense? 2) How one can analyze the coverage and rate for less dense cellular networks? Because, when it comes to practical situations not all regions need to be densely deployed. Since the requirements vary from region to region, for example, the traffic patterns in residential regions would definitely be different from commercial ones. 3) How much intensity of BSs belonging to certain tier is required to provide minimum coverage and rate in a given region? Thus, the prime motivation of this research is to explore answers of the above mentioned questions by incorporating some factors into existing stochastic geometry model [7] for single-tier cellular network. The main contributions of this work are listed as under.

- A modified model for coverage analysis of single-tier cellular network by exploiting tools from stochastic geometry. The presented model is not independent of intensity or transmitted power of tiers; hence, it is equally applicable to both dense and sparse networks.

- The intensity of BSs for Pico and Femto tiers has been approximated through simulations, such that they can at least meet the coverage bounds given in [7].

- Monte-Carlo (MC) simulations have been performed for validation of proposed approach and results are compared at the end in Section IV.

\section{System Model}

In this work we assume a single-tier cellular network, where the location of base stations (BSs) has been drawn from a Poisson point process (PPP) $\boldsymbol{\Phi}$ of intensity $\boldsymbol{\lambda}$. The mobile stations (MSs) i.e., customers, are assumed to be distributed according to another independent PPP $\boldsymbol{\Phi}_{\mathbf{u}}$ with intensity $\boldsymbol{\lambda}_{\mathbf{u}}$. Nearest BS association rule and downlink channel have been considered under this study. Thus, a customer is said to be under coverage if received signal to interference noise ratio (SINR) exceeds some pre-defined threshold $\theta$. Where, SINR can be defined as,

$$
\operatorname{SINR}\left(x_{o}\right)=\frac{P_{r}\left(x_{o}\right)}{\sum_{x \in \Phi_{c} \backslash x_{o}} P_{r}(x)+\sigma^{2}}
$$

here, $\boldsymbol{P}_{\boldsymbol{r}}$ in numerator denotes the amount of power received from tagged $\mathrm{BS}$ and in denominator, the sum over it, represents the amount of received power from all interfering BSs. " $\boldsymbol{\sigma}$ " denotes noise power, which is assumed to be additive and constant. The amount of power received $\boldsymbol{P}_{\boldsymbol{r}}(\boldsymbol{x})$ at a randomly located mobile user from a BS $2 \Phi$, is actually function of three factors as given in (2).

$$
P_{r}(x)=P_{t} \cdot h_{x} \cdot l(x)
$$

1) The amount of power transmitted $\boldsymbol{P}_{\boldsymbol{t}}, 2$ ) Random fading component $\boldsymbol{h}_{\boldsymbol{x}} \sim \exp (\boldsymbol{\mu})$, that is assumed to be exponentially distributed with mean 1 over the link between MS and tagged BS. For Interfering links, it follows a general distribution " $g$ " however, closed form expressions can be obtained when fading over interfering links also follow exponential distribution. 3) Distance dependent path loss component $\boldsymbol{l}(\boldsymbol{x})$, which has been defined by considering free space path loss (FSPL) model with reference distance of $1 \mathrm{~m}$ and given as $\boldsymbol{l}(\boldsymbol{x})=\left(\frac{\lambda_{w} \cdot l}{4 \pi}\right)^{2} \boldsymbol{x}^{-\alpha}$. Here, $\lambda_{w}$ is wavelength of operating channel, $\boldsymbol{\alpha}>\mathbf{2}$ is the path loss exponent. 


\section{Proposed Approach}

According to [7]-[9], an MS is said to be under coverage if it receives a target SINR, $\boldsymbol{\theta}$. According to [15], in modern cellular networks such as LTE, the threshold for received signal power $\left(\boldsymbol{P}_{\boldsymbol{r}}\right)$ is low enough to be comfortably exceeded by any modern receiver within a fairly large distance from transmitting BS. Therefore, for the purpose of analysis $\boldsymbol{P}_{\boldsymbol{r}}$ can be dropped and without loss of generality only SINR can be considered key indicator of coverage, as given in eq. (3). Thus, an MS is said to be under coverage, if communication link from the BS serving that MS is sufficiently good; where good means $\boldsymbol{S I N R}>\boldsymbol{\theta}$. This definition for coverage analysis is very suitable when considering dense networks. However, this is not always the case; as some regions are less densely deployed as compared to the others due to variation in traffic patterns and intensity of customers i.e., $\lambda_{\boldsymbol{u}}$.

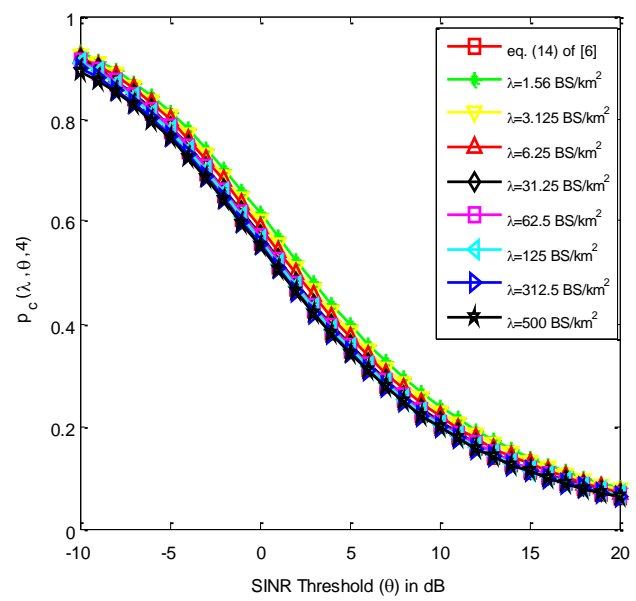

Figure 1: $\mathrm{MC}$ simulation results as a function of $\lambda$, [7]

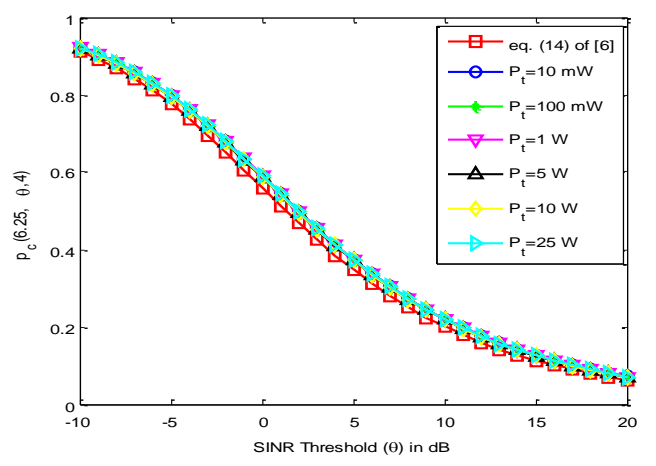

Figure 2: MC simulation results as a function of $P_{t},[7]$

Therefore, while considering less dense networks for certain regions; as distance between MS and tagged BS exceeds certain threshold $x>R$, the received signal strength $\boldsymbol{P}_{r}$ drops below defined threshold $\delta$. Hence, MS can no more be considered under coverage. Since SINR is a ratio, it will remain the same either for dense or sparse networks. That's the reason, it does not get affected by intensity of BSs deployed, as discussed in [7]. Thus, for better insight into different parameters of interest from coverage and capacity point of view, it is important to consider the effect of $\boldsymbol{P}_{\boldsymbol{r}}$ along with SINR as defined in eq. (4).

$$
\begin{gathered}
\boldsymbol{p}_{c}(\lambda, \theta, \alpha)=P[S I N R>\theta] \\
p_{c}\left(\lambda, \theta, \delta, P_{t}\right)=P[S I N R>\theta \mid x<R]
\end{gathered}
$$

(4)

Without loss of generality, we consider that MS is located at origin and the nearest BS is at a distance $\boldsymbol{x}$ from it; such that $\boldsymbol{x}<\boldsymbol{R}$ which makes sure that $\boldsymbol{P}_{\boldsymbol{r}}>\boldsymbol{\delta}$. Hence, MS is said to be under coverage if it receives SINR greater than $\theta$.

$$
\operatorname{SINR}\left(x_{o}\right)=\frac{P_{r}\left(x_{o}\right)}{I_{x}+\sigma^{2}}
$$

where, $\boldsymbol{I}_{\boldsymbol{x}}=\sum_{\boldsymbol{x} \in \boldsymbol{\Phi}_{\boldsymbol{c}} \backslash \boldsymbol{x}_{\boldsymbol{o}}} \boldsymbol{P}_{\boldsymbol{r}}(\boldsymbol{x})$ is the cumulative interference from all BSs $2 \Phi$ other than tagged one. Since nearest BS association rule has been assumed, the probability density function (PDF) for distance $x$ between MS and 
tagged BS can be given as $\boldsymbol{f}_{\boldsymbol{x}}(\boldsymbol{x})=$ $2 \pi \boldsymbol{\lambda} \boldsymbol{x} \boldsymbol{e}^{-\boldsymbol{\pi} \lambda x^{2}}$. The maximum hard core distance $R$ beyond which an MS cannot be considered under coverage has been obtained from eq. (2) as,

$$
R=\left(\frac{P_{t} \cdot \mu \cdot K}{\delta}\right)^{1 / \alpha}
$$

Where, $\boldsymbol{K}=\left(\frac{\lambda_{w} \cdot l}{4 \pi}\right)^{2}$ and $\mu$ is mean of random fading variable, $\boldsymbol{h}_{\boldsymbol{x}}$, which is exponentially distributed.

\subsection{General Case}

The general case results for coverage analysis of single-tier network have been presented in this section.

Theorem 1: The probability that an MS will be under coverage of cellular network with parameters described in Section II is,

$$
\begin{aligned}
& p_{c}\left(\lambda, \theta, \alpha, \delta, P_{t}\right) \\
& =2 \pi \lambda \int_{0}^{R} e^{-\pi \lambda x^{2} \beta(\theta, \alpha)-\mu \theta \sigma^{2} x^{\alpha / 2}} x d x
\end{aligned}
$$

where,

$$
\begin{gathered}
\beta(\theta, \alpha)=\frac{2(\mu \theta)^{2 / \alpha}}{\alpha} \mathrm{E}\left[g ^ { 2 / \alpha } \left\{\Gamma\left(-\frac{2}{\alpha}, \mu \theta g\right)\right.\right. \\
\left.\left.-\Gamma\left(-\frac{2}{\alpha}\right)\right\}\right]
\end{gathered}
$$

here, expectation is with respect to, $g$, channel fading distribution of interfering BSs. $\boldsymbol{\Gamma}(\boldsymbol{a}, \boldsymbol{x})=\int_{x}^{\infty} \boldsymbol{z}^{\boldsymbol{a}-\mathbf{1}} \boldsymbol{e}^{-\boldsymbol{z}} \boldsymbol{d z}$ denotes incomplete gamma function, and $\Gamma(x)=\int_{0}^{\infty} z^{x-1} e^{-z} d z$ is the standard gamma function.

Proof: By conditioning on the fact that, nearest BS is located at $x$ distance from a randomly located MS such that $x<\boldsymbol{R}$, probability of coverage averaged over the plane can be given as,

$$
\begin{aligned}
& \boldsymbol{p}_{c}\left(\lambda, \theta, \alpha, \delta, P_{t}\right)=E_{x}[P\{S I N R>\theta \mid x \\
&<R\}] \\
&=\int_{x>0} P[S I N R>\theta \mid x<R] . f_{x}(x) d x
\end{aligned}
$$

$$
\begin{aligned}
& =\int_{0}^{R} P\left[\frac{h_{x} x^{-\alpha}}{I_{x}+\sigma^{2}}\right] \cdot f_{x}(x) d x \\
& =\int_{0}^{R} P\left[h_{x}>\theta x^{\alpha}\left(I_{x}+\sigma^{2}\right)\right] \cdot f_{x}(x) d x
\end{aligned}
$$

Since probability of coverage for $\boldsymbol{x}>\boldsymbol{R}$ is zero, the limit of integral has been extended from 0 to $R$ instead of 0 to 1 , which is generally considered for dense cellular networks. Since it has been assumed that $\boldsymbol{h}_{\boldsymbol{x}} \sim \boldsymbol{\operatorname { e x p }}(\boldsymbol{\mu})$, the probability that random fading component $\boldsymbol{h}_{\boldsymbol{x}}$ has value greater than required can be obtained as,

$$
\begin{aligned}
P\left[h_{x}>\right. & \left.\theta x^{\alpha}\left(I_{x}+\sigma^{2}\right) \mid x\right]= \\
& E_{I_{x}}\left[P\left\{h>\theta x^{\alpha}\left(I_{x}+\sigma^{2}\right) \mid x, I_{x}\right\}\right] \\
& =E_{I_{x}}\left[\exp \left\{-\mu \theta x^{\alpha}\left(I_{x}+\sigma^{2}\right)\right\}\right] \\
& =e^{-s \sigma^{2}} \mathcal{L}_{I_{x}}(s)
\end{aligned}
$$

where, $\boldsymbol{s}=\boldsymbol{\mu} \boldsymbol{\theta} \boldsymbol{x}^{\boldsymbol{\alpha}}$ and $\boldsymbol{L}_{\boldsymbol{I}_{\boldsymbol{x}}}(\boldsymbol{s})$ is Laplace transform of random variable $\boldsymbol{I}_{\boldsymbol{x}} x$ evaluated at $s$, by conditioning on the fact that, MS

located at origin associates with nearest BS. Thus probability of coverage can be obtained as,

$$
\begin{aligned}
& p_{c}\left(\lambda, \theta, \alpha, \delta, P_{t}\right)= \\
& 2 \pi \lambda \int_{0}^{R} e^{-s \sigma^{2}} \mathcal{L}_{I_{x}}(s) e^{-\pi \lambda x^{2}} x d x
\end{aligned}
$$

For general fading distribution, the Laplace transform of interference for singletier cellular network with BS locations drawn from PPP has been derived in [6] as,

$$
\mathcal{L}_{I_{x}}(s)=e^{-\pi \lambda x^{2}\{\beta(\theta, \alpha)-1\}}
$$

Thus substituting eq. (7) in eq. (6) proves the theorem.

\subsection{Special Case: General Fading, Interference Limited Network}

The assumption for interference limited regime is frequently considered in literature [7]-[9], due to the fact that, in modern cellular networks thermal noise power is negligible as compared to desired signal power; while 
considering interior of cells. On the other hand, at cell edges the interference is typically much larger than thermal noise. Hence for simpler expressions it is preferred to consider interference limited regime i.e., $\sigma 2=0$. With this assumption, Theorem 1 will be simplified as,

$p_{c}\left(\lambda, \theta, \alpha, \delta, P_{t}\right)=\frac{1-e^{-\pi \lambda R^{2} \beta(\theta, \alpha)}}{\beta(\theta, \alpha)}$

It must be noted here that, as $\boldsymbol{\lambda}$ or $\boldsymbol{P}_{\boldsymbol{t}}$ approaches 1 , the exponential term in eq. (8) approaches zero. The resulting expression becomes independent of $\boldsymbol{\lambda}$ or $\boldsymbol{P}_{\boldsymbol{t}}$ of chosen tier and becomes equivalent to one given in eq.(8) of [7], for dense interference limited cellular network. Thus, with the help of expressions in eq. (8) we can estimate the limit of $\lambda$ for particular tier with transmitted power $\boldsymbol{P}_{\boldsymbol{t}}$, beyond which it can be considered as dense and no further improvements can be achieved in $\boldsymbol{p c}$.

\subsection{Special Case: Rayleigh Fading, Interference Limited Network}

Simplified expression can be obtained when fading on interfering links is also assumed to be exponentially distributed. In Theorem 2 of [7], the Laplace transform of interference while considering Rayleigh fading has been derived and resulting expression is given as,

$$
\mathcal{L}_{I_{x}}(s)=e^{-\pi \lambda x^{2} \rho(\theta, \alpha)}
$$

where,

$$
\rho(\theta, \alpha)=\theta^{2 / \alpha} \int_{\theta^{2 / \alpha}}^{\infty} \frac{1}{1+u^{\alpha / 2}} d u
$$

Thus, plugging eq. (9) into eq. (6), results in expression of coverage for a randomly located MS experiencing exponential fading on all links as,

$$
\begin{aligned}
& p_{c}\left(\lambda, \theta, \alpha, \delta, P_{t}\right)= \\
& 2 \pi \lambda \int_{0}^{R} e^{-\pi \lambda x^{2}\{1+\rho(\theta, \alpha)\}-\mu \theta \sigma^{2} x^{\alpha / 2}} x d x(10)
\end{aligned}
$$

For no noise case i.e., $\boldsymbol{\sigma}^{\mathbf{2}}=\mathbf{0}$, the resulting expression will be further simplified as,

$$
p_{c}\left(\lambda, \theta, \alpha, \delta, P_{t}\right)=\frac{1-e^{-\pi \lambda R^{2}\{1+\rho(\theta, \alpha)\}}}{1+\rho(\theta, \alpha)}
$$

where, $\boldsymbol{\rho}(\boldsymbol{\theta}, \boldsymbol{\alpha})$ is much easier and faster to compute as compared to $\boldsymbol{\beta}(\boldsymbol{\theta}, \boldsymbol{\alpha})$. By further assuming $\alpha=4$, a complete closed form expression for $\boldsymbol{\rho}(\boldsymbol{\theta}, \mathbf{4})$ can be obtained as,

$$
\rho(\theta, 4)=\sqrt{\theta}\left\{\pi / 2-\tan ^{-1}\left(\frac{1}{\sqrt{\theta}}\right)\right\}
$$

Thus, substituting eq. (12) into eq. (11) results in final expression for probability of coverage as,

$p_{c}\left(\lambda, \theta, \alpha, \delta, P_{t}\right)=\frac{1-e^{-\pi \lambda R^{2} \sqrt{\theta}\left\{\pi / 2^{-\tan ^{-1}}\left(\frac{1}{\sqrt{\theta}}\right)\right\}}}{1+\sqrt{\theta}\left\{\pi / 2^{\left.-\tan ^{-1}\left(\frac{1}{\sqrt{\theta}}\right)\right\}}\right.}$

The obtained expression in eq. (13) is very simple and approaches to one, given in eq. (14) of [7], as $\boldsymbol{\lambda}$ or $\boldsymbol{P}_{\boldsymbol{t}}$ approaches to 1 .

\section{Results and Discussions}

For tiers like Femto or Pico, the assumption of PPP is directly valid but the deployment of Macro BSs involve planning; hence, cannot be considered as completely random. That's why in [7], [8], it has been justified that PPP though exhibits the property of complete spatial randomness; however, provides pessimistic bounds for coverage and rate analysis of dense Macro-tier. In this research authors suggest that, it is also equally valid to consider PPP for sparse Macro-tier. With significant decrease in $\lambda$, the probability that, two BSs are close to each other approaches to zero. Thus, resulting expressions becomes free of high interference issues from nearby BSs. 


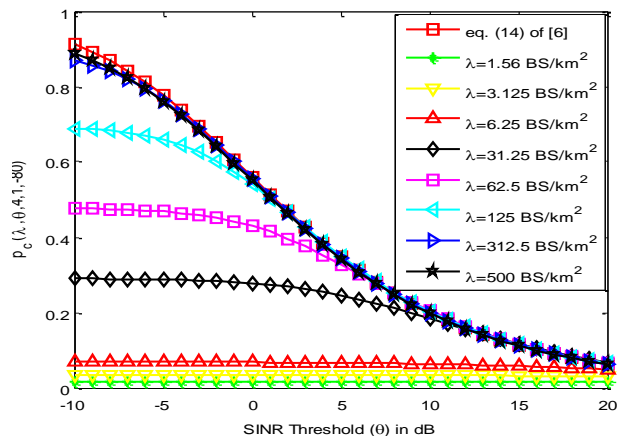

Figure 3: MC simulation results as a function of $\boldsymbol{\lambda}$, proposed approach

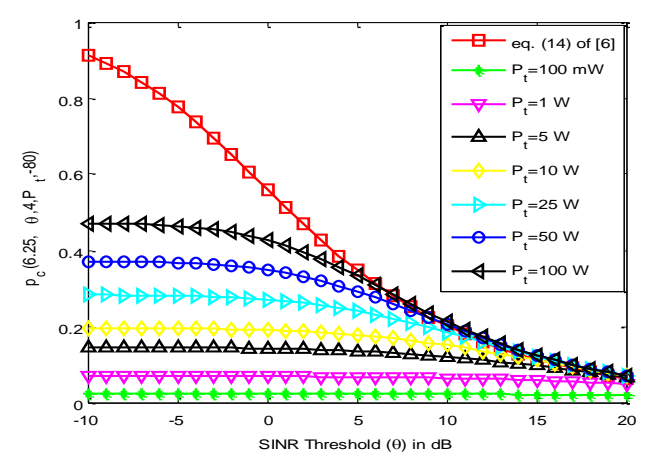

Figure 4. MC simulation results as a function of $P_{t}$, proposed approach

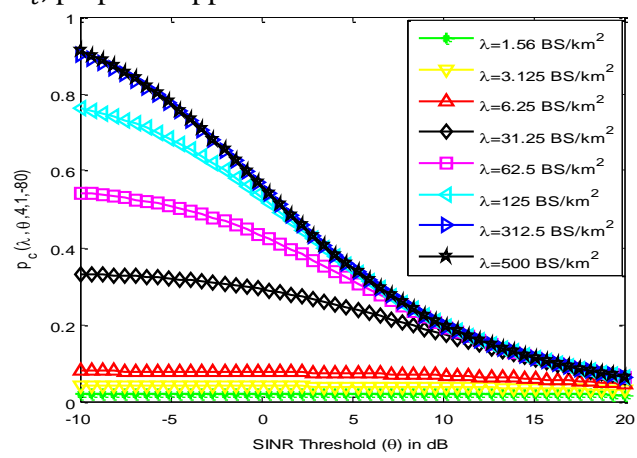

Figure 6. Analytical results as a function of $\lambda$, proposed approach

Similarly, while considering practical situations, though the deployment of Macro BSs is planned; however, a constant distance as supposed in grid models [5] cannot be maintained due to various issues like availability of space, terrain conditions, traffic requirements and others. Even though, there is not complete spatial randomness as supposed by PPP, but to certain extent it does involve randomness. Thus, the location of BSs for sparse networks can also be approximated by PPP as in the case of dense networks. In [7], the authors have already compared the results of stochastic geometry based approach with grid model. Therefore, in this research, we have performed Monte-Carlo (MC) simulations. The results are compared with analytical expressions obtained in this work and one presented in [7].

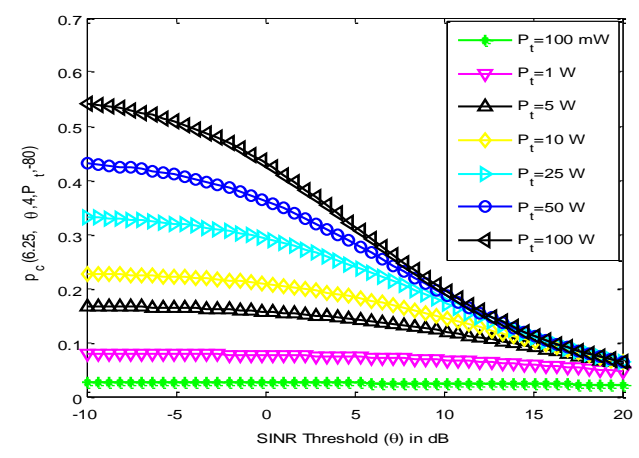

Figure 5. Analytical results as a function of $P_{t}$ proposed approach

\subsection{Monte-Carlo Simulations}

For comparison of results, we realized the network described in Section II through Monte-Carlo (MC) simulations. An area of $\mathbf{4} * \mathbf{4} \mathbf{k m}^{2}$ was considered and BSs of intensity $\lambda$, with identical power of transmission $\boldsymbol{P}_{\boldsymbol{t}}$, were randomly placed in considered region using uniform distribution. The distance between an MS and all BSs were measured by using expression, $\boldsymbol{x} \boldsymbol{n}=$ $\sqrt{\left(x_{n}-x_{o}\right)^{2}+\left(y_{n}-y_{o}\right)^{2}}$ where, $x_{o} \&$ $\boldsymbol{y}_{\boldsymbol{o}}$ represent coordinates of MS location and $\boldsymbol{x}_{\boldsymbol{n}} \& \boldsymbol{y}_{\boldsymbol{n}}$ denote coordinates for $\boldsymbol{n}^{\text {th }}$ BS location. For every MS location, the BS with $\min \left(x_{n}\right)$ was selected as tagged one and rest were treated as interfering BSs. Thus, SINR at every MS location was calculated by using eq. (1) with $\boldsymbol{\sigma}^{2}=\mathbf{0}$ and results were averaged down at the end for obtaining probability of 
coverage. Each simulated average result has been obtained by repeating 1000 iterations. It must be noted that all MC simulations have been performed for interference limited network with $\boldsymbol{\alpha}=\mathbf{4}, \boldsymbol{f} \boldsymbol{c}=1800 \mathrm{MHz}, \boldsymbol{\lambda}$ in BS $/ \mathrm{km} 2, \boldsymbol{\delta}$ in dBm, $\mu=\mathbf{1}$, and $\boldsymbol{P}_{\boldsymbol{t}}$ in watts, if specifically not mentioned.

\subsection{Results for MC Simulations}

Initially for a better insight into results, MC simulations have been performed. Coverage definition given in eq. (3) has been considered, which is extensively used in literature [7]-[9], [15]. It must be noted from MC results given in Fig. 1 and 2 that, no matter how sparse or dense network we chose, the resulting SINR distribution is similar to that proposed in [7]. Since SINR is a ratio, thus while considering sparse or dense networks the received signal strength from tagged and interfering BSs are affected by almost same amount. Therefore, in each case MC simulation produces same results. That's the reason that, under especial cases, $\boldsymbol{p}_{\boldsymbol{c}}$ for multi-tier proposed in [8], [9] is the same as that for single-tier given in [7].

MC simulations have also been performed by redefining the $p c$, as proposed in this research and given in eq. (4), under the same scenario. In Fig. 3, the probability of coverage i.e., eq. (4), has been plotted as a function of BS density. It must be interesting to note that, by varying $\boldsymbol{\lambda}, \boldsymbol{p}_{\boldsymbol{c}}$ is changing and after increasing $\boldsymbol{\lambda}$ to certain level, $\boldsymbol{p}_{\boldsymbol{c}}$ does not vary and approaches to limit given in [7]. Similarly, in Fig. 4 probability of coverage has been plotted against BSs operating at different power levels. It must be clear that, BSs operating at higher power level provides better coverage as compared to ones operating at lower power levels. It is obvious; Macro BSs provide better coverage as compared to Femto or Pico BSs.

\subsection{Analytical Results of Proposed Approach}

Here we discuss main results of the proposed approach. As already clear from results of MC simulations that by following eq. (4), we can have better insight into coverage analysis of cellular networks. In Fig. 5 and 6 , the closed form expressions obtained in eq. (13) have been plotted. As we increase the density of BS the coverage probability improves. It must be clear that proposed framework provides almost the same results as given in Fig. 3 for MC simulations. Similarly, in Fig. 6, the coverage probability has been plotted against transmitted power of BS by exploiting proposed analytic expression given in eq. (13). It must be clear that, coverage probability improves in proportion with transmitted power of BSs and is comparable with results given in Fig. 4. Thus, this verifies the validity of proposed approach. However, the proposed analytical expressions provide a bit over estimation for $\boldsymbol{p}_{\boldsymbol{c}}$ as compared to results obtained through MC simulations. For example, in Fig. 3 and 5, for $\lambda=125 \mathrm{BS} /$ $\mathbf{k m}^{2}$ compare two curves. In Fig. 3, at $\boldsymbol{S I N R}=-\mathbf{1 0 d B}, \boldsymbol{p}_{\boldsymbol{c}} \approx \mathbf{0}$. 7. However, in Fig. 4, at $\boldsymbol{S I N R}=-\mathbf{1 0 d B}, \boldsymbol{p}_{\boldsymbol{c}} \approx \mathbf{0} . \mathbf{7 5}$, but it is as per expectation. Since, we assumed hard core distance $R$ for received signal strength which does not accounts for irregular boundary effects.

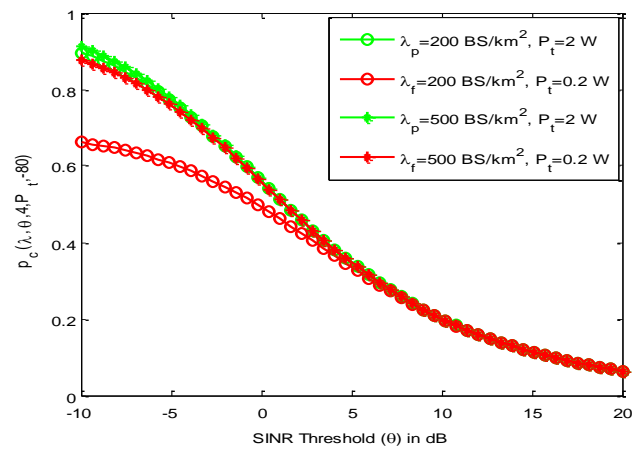

Figure 7. Estimating intensity of tiers i.e., $\lambda$ for $\delta=-80 \mathrm{dBm}$ 


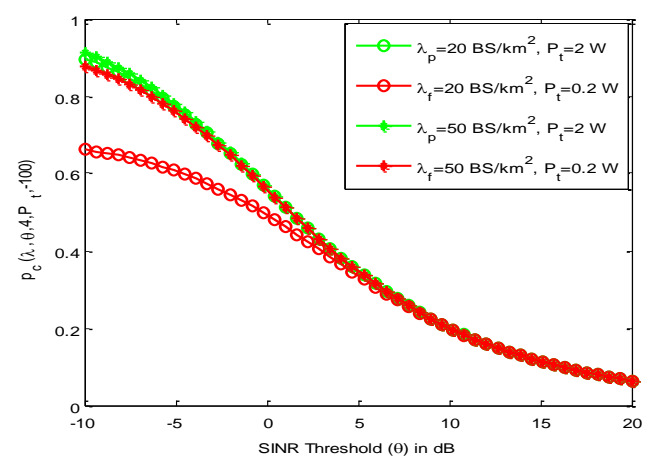

Figure 8. Estimating intensity of tiers i.e., $\lambda$ for $\delta=-100 \mathrm{dBm}$

On the other hand in Fig. 8, the results are obtained by assuming $\boldsymbol{\delta}=-\mathbf{1 0 0 d B m}$, which is fair $\boldsymbol{P}_{r}$ in cellular network. As hard core distance $R$ is increased in the case of $\boldsymbol{\delta}=$ $-100 \mathrm{dBm}$, therefore $\lambda$ for each tier has been decreased. Another, important fact to note here is that, $\lambda_{p}<\lambda_{f}$ since $\boldsymbol{P}_{\boldsymbol{t}}$ for Pico tier is higher as compared to Femto. After increasing $\lambda$ behind certain limit (i.e., in Fig. $8, \lambda_{p}>$ 20) no further improvements in $\boldsymbol{p}_{\boldsymbol{c}}$ has been achieved and it never exceeds the bounds defined in [7]. Thus, the proposed approach is equally valid for sparse as well as for dense networks. It is more important for industry practitioners, since they can tune the parameters of interest as per intensity of customers and traffic patterns in certain region under consideration.

\section{Conclusion}

A modified stochastic geometry based tractable approach has been presented in this research, which takes into account the effect of received signal strength in addition to SINR. With the help of simple modification into existing definition for probability of coverage, parameters of interest i.e., $\boldsymbol{\lambda}$ and $\boldsymbol{p}_{\boldsymbol{c}}$ can be estimated for different tiers of cellular network under different scenarios. Various results have been reported where the effect of BS density and power of transmission on coverage probability has been analyzed in detail. Apart from that, the density of different tiers has also been estimated under different received signal strength thresholds. Although the work presented provides just an initial insight into the effects of considering a modified definition for $\boldsymbol{p}_{\boldsymbol{c}}$, by limiting the coverage radius for single-tier cellular network to hard core distance $R$. However, more accurate models can be obtained by considering joint probabilistic definition for $\boldsymbol{p}_{\boldsymbol{c}}$ or by using other point processes, which consider the effect of minimum distance or repulsion into account. For validation of proposed approach and its comparison with existing results, Monte-Carlo simulations have been performed. It has been concluded that, after certain limit for $\boldsymbol{\lambda}$ or $\boldsymbol{P}_{\boldsymbol{t}}$, the results of proposed approach becomes equivalent to one presented in [7] for single-tier dense cellular network.

\section{REFERENCES}

[1] A. Ghosh, N. Mangalvedhe, R. Ratasuk, B. Mondal, M. Cudak, E. Visotsky, T. A. Thomas, J. G. Andrews, P. Xia, H. S. Jo et al., "Heterogeneous cellular networks: From theory to practice," IEEE Communications Magazine, vol. 50, no. 6, pp. 54-64, 2012.

[2] W. H. Chin, Z. Fan, and R. Haines, "Emerging technologies and research challenges for $5 \mathrm{~g}$ wireless networks," IEEE Wireless Communications, vol. 21, no. 2, pp. 106-112, 2014.

[3] J. G. Andrews, S. Buzzi, W. Choi, S. V. Hanly, A. Lozano, A. C. Soong, and J. C. Zhang, "What will 5G be?," IEEE Journal on selected areas in communications, vol. 32 , no. 6 , pp.10651082, 2014.

[4] S. Shamai and A. D. Wyner, "Information-theoretic considerations for symmetric, cellular, multiple-access fading channels. i \& ii," IEEE Transactions on Information Theory, vol. 43, no. 6, pp. 1877-1894,1997.

[5] T. X. Brown, "Cellular performance bounds via shotgun cellular systems," IEEE Journal on Selected Areas in 
Communications, vol. 18, no. 11, pp. 2443-2455, 2000.

[6] J. Xu, J. Zhang, and J. G. Andrews, "When does the wyner model accurately describe an uplink cellular network?" in 2010 IEEE Global Telecommunications Conference GLOBECOM 2010, Dec 2010, pp. 1-5.

[7] J. G. Andrews, F. Baccelli, and R. K. Ganti, "A tractable approach to coverage and rate in cellular networks," IEEE Transactions on Communications, vol. 59, no. 11, pp. 3122-3134, 2011.

[8] H. S. Dhillon, R. K. Ganti, F. Baccelli, and J. G. Andrews, "Modeling and analysis of k-tier downlink heterogeneous cellular networks," IEEE Journal on Selected Areas in Communications, vol. 30, No. 3, pp. 550-560, April 2012.

[9] H.-S. Jo, Y. J. Sang, P. Xia, and J. G. Andrews, "Heterogeneous cellular networks with flexible cell association: A comprehensive downlink SINR analysis," IEEE Transactions on Wireless Communications, vol. 11, no. 10, pp. 3484-3495, 2012.

[10] Y. Li, F. Baccelli, H. S. Dhillon, and J. G. Andrews, "Statistical modeling and probabilistic analysis of cellular networks with determinantal point processes," IEEE Transactions on Communications, vol. 63, no. 9, pp. 3405-3422, 2015.

[11] Y. J. Chun, M. O. Hasna, and A. Ghrayeb, "Modeling heterogeneous cellular networks interference using poisson cluster processes," IEEE Journal on Selected Areas in Communications, vol. 33, no. 10, pp. 2182-2195, Oct 2015.

[12] N. Deng, W. Zhou, and M. Haenggi, "Heterogeneous cellular network models with dependence," IEEE Journal on Selected Areas in Communications, vol. 33, no. 10, pp. 2167-2181, 2015.

[13] J. G. Andrews, X. Zhang, G. D. Durgin, and A. K. Gupta, "Are we approaching the fundamental limits of wireless network densification?," IEEE
Communications Magazine, vol. 54 no. 10, pp.184-190, 2016

[14] X. Zhang, and J. G. Andrews, "Downlink cellular network analysis with multislope path loss models," IEEE Transactions on Communications, vol. 63, no. 5, pp.1881-1894, 2015

[15]S. Mukherjee, Analytical Modeling of Heterogeneous Cellular Networks. Cambridge University Press, 2014. 